\title{
Heterogeneity and lateral compartmentalization of plant plasma membranes
}

\author{
Nana Friderike Zappel and Ralph Panstruga
}

\begin{abstract}
Membrane specialization through lateral compartmentalization is pivotal to the development of organisms and their response to environmental signals. The membrane raft hypothesis is lively discussed as a concept for domain formation. In recent years plant scientists have begun to critically assess the membrane raft hypothesis, and this provided the first insights into the mechanisms underlying microdomain formation in plant plasma membranes. Several groups have now shown that phytosterols can induce phase separation, a prerequisite for the formation of membrane rafts. Furthermore, the protein repertoire of detergent-resistant membranes (DRMs) has been extensively characterized and the degree of fatty acid desaturation has been identified as an important factor in DRM formation. Recent studies comprising sterol-deficient mutants demonstrated the importance of correct sterol composition and endocytosis for proper membrane compartmentalization.
\end{abstract}

\author{
Address \\ Max Planck Institute for Plant Breeding Research, Department of Plant \\ Microbe Interactions, Carl-von-Linné-Weg 10, 50829 Cologne, Germany \\ Corresponding author: Panstruga, Ralph (panstrug@mpiz-koeln.mpg.de) \\ Current Opinion in Plant Biology 2008, 11:632-640 \\ This review comes from a themed issue on \\ Cell Biology \\ Edited by David Ehrhardt and Federica Brandizzi \\ Available online 4th September 2008 \\ $1369-5266 / \$$ - see front matter \\ (C) 2008 Elsevier Ltd. All rights reserved. \\ DOI 10.1016/j.pbi.2008.07.002
}

\section{Introduction}

Since Singer and Nicolson proposed their fluid mosaic model on biological membranes [1] our picture of such membranes has considerably evolved. Today, it is accepted that membranes are highly organized structures providing the necessary heterogeneity to compartmentalize cellular processes [2]. It is plausible that compartmentalization and thereby specialization of cellular processes at the level of biological membranes is essential for the development of organisms and their response to environmental signals. To achieve this membrane specialization, a lateral organization of biological membranes is required. However, so far there is no consensus on the underlying molecular principles and mechanisms driving lateral heterogeneity [3]. The membrane raft hypothesis is a conceivable explanation for the spatial and temporal organization of membranes [4]. Membrane rafts are thought to form distinct domains within the lipid bilayer through the tight interaction of sphingolipids and sterols. Proteins are assumed to be specifically included or excluded from membrane rafts, thereby providing a mechanism for confined protein clustering [3]. The membrane raft hypothesis is based on the phase behavior of model membranes, in which liquid ordered $\left(l_{0}\right)$ and liquid disordered $\left(l_{d}\right)$ phases can coexist. Sterols have the ability to drive the formation of $1_{o}$ phases; however, the precise mechanism of phase separation is unclear [5]. The controversy about the membrane raft hypothesis arises from observations of macroscopically visible coexisting phases in model membranes but not in resting (non-stimulated) living cells. Yet strong evidence has accumulated regarding the existence of very small and highly dynamic $l_{0}$ domains in biological membranes [2]. This led to the revised definition of membrane rafts as "small (10$200 \mathrm{~nm}$ ), heterogeneous, highly dynamic, sterol-enriched and sphingolipid-enriched domains that compartmentalize cellular processes. Small rafts can sometimes be stabilized to form larger platforms through proteinprotein and protein-lipid interactions" [6]. To finally help resolve the open questions concerning size, composition, stability, mechanism of formation, and physical properties of membrane rafts, new technologies and concepts will probably be needed [2,3,7]. At the same time, researchers mainly agree that whether small membrane rafts preexist or not, large-scale phase separation can occur through either clustering of preexisting rafts or the stabilization and coalescence of transient rafts $([7,8]$; Figure 1). Examples for such induced large-scale clustering in eukaryotic cells are the vertebrate immunological synapse $[9,10]$, projection sites of mating yeast cells [11,12], cell adhesion sites [13], and the tips of growing hyphae in Candida albicans [14]. In addition to the membrane raft hypothesis the meshwork hypothesis also explains the compartmentalization of plasma membranes (PMs). In this model it is assumed that the cortical actin cytoskeleton (so called fences) and transmembrane proteins anchored to it (so-called pickets) restrict the lateral diffusion of transmembrane proteins [15]. Although the latter model does not account for the compartmentalization of lipids, it is conceivable that the lateral mobility of (trans-) membrane proteins is modulated on the basis of both lipid-dependent and actin-dependent organization forces [16].

In plant research, membrane domains and dynamics, cell polarity and the role of lipids and sterols therein have gained increasing attention in the past few years. Cell 


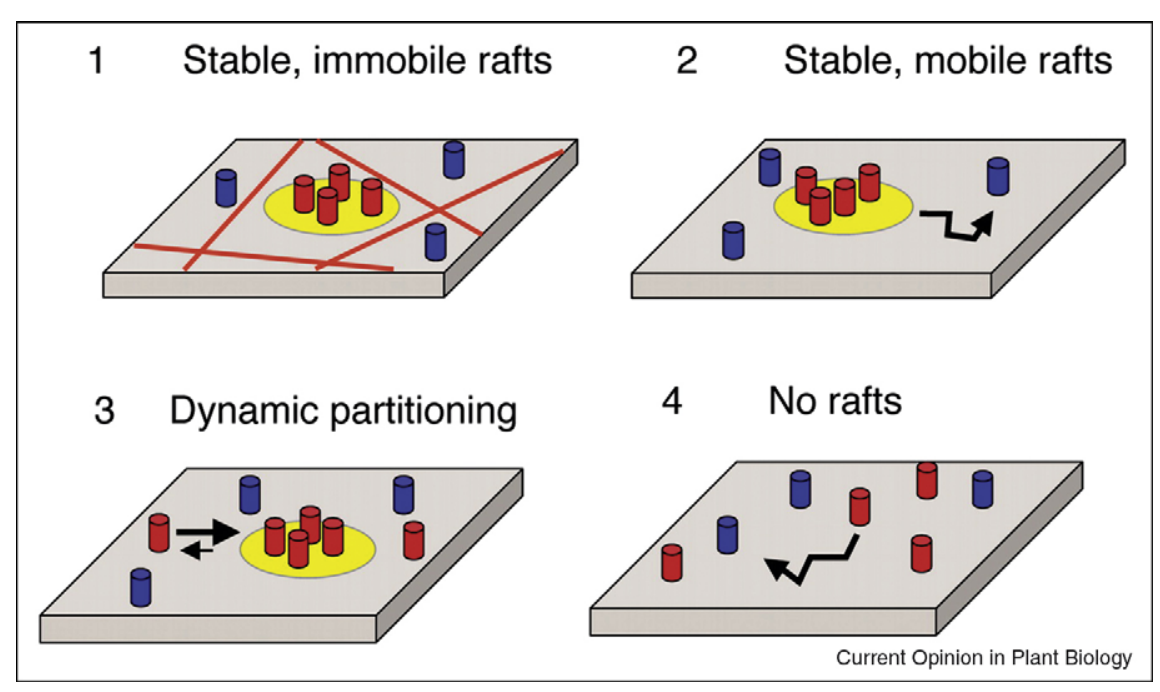

Models for membrane raft dynamics. Models for diffusional mobility of membrane rafts (yellow), raft-associated proteins (red), and nonraft proteins (blue). (1) Stable, immobile rafts. Hypothetical barriers to membrane raft diffusion are depicted by red lines. (2) Stable, mobile rafts. (3) Dynamic partitioning of raft proteins. (4) No rafts. For simplicity, putative barriers to individual protein diffusion are not depicted. Copyright 2004 [8], published by The Rockefeller University Press.

polarization and membrane domain formation are indispensable for plants since they rely on cellular and subcellular asymmetry during development. Polar growth of the pollen tube and root hairs depend on cell polarization as well as transporters that are focally localized in membrane domains and that seem to be regulated thereby $\left[17,18,19^{\bullet}, 20,21^{\bullet}\right]$. Furthermore, plasticity and the ability to polarize the whole cell including membrane proteins is needed for the plant cells' response to environmental stimuli, such as pathogen attack [22-25]. Since the mechanisms underlying these membrane polarizations are still largely unknown it will be interesting to investigate the possible involvement of membrane rafts in plant membrane heterogeneity. Membrane raft research in plants may help to substantiate key findings obtained using animal systems but could also open up new possibilities of critically testing the membrane raft hypothesis by employing forward and reverse genetic approaches in a multicellular organism. Here, we review the recent findings on membrane domains and cell polarity in plants with a special focus on the possible role for membrane rafts in various biological processes.

\section{Model membranes}

In contrast to animal membranes where cholesterol is the predominant sterol, plant membranes comprise a more complex sterol mixture. The main sterols in Arabidopsis cells are sitosterol, stigmasterol, and campesterol, while cholesterol contributes only a minor proportion. One of the basic prerequisites for the existence of membrane rafts in plant cells is the induction of phase separation by phytosterols. The ability of sterols to pack tightly with saturated lipids is the key for their domain-forming activity [26]. To date, several groups described the domain-promoting activity of phytosterols in two-component and three-component lipid mixtures. They have shown that the order of enriched domains and their stability depends on the structure of the partitioning sterol [27-31]. However, probably owing to differences in experimental systems and their methods, no clear ranking of the sterols concerning their domain-forming ability, domain-stabilizing activity, or the order of the induced domains has been established. Beck et al. demonstrated that the dynamics of three-component mixtures resembling plant membranes are less sensitive to temperature changes than mixtures mimicking animal or fungal membranes [30]. The domain stability over a wider temperature range suggests that plants evolved a superior mechanism to cope with changing temperatures, ensuring proper functioning of membrane-associated processes. This feature is possibly one of the many adaptations of plants to their sessile lifestyle.

\section{Detergent-resistant membranes}

Brown and Rose introduced the concept of membrane rafts being resistant to detergent treatment based on the tight interaction of lipids, proteins, and cholesterol [32]. During treatment the detergent molecules insert preferentially into the $l_{d}$ phase. Above a certain detergent concentration the $l_{d}$ phase solubilizes leaving the $l_{o}$ phase intact. Owing to these characteristics and its practicability, detergent-resistant membrane (DRM) isolation is probably the method not only most widely used to study membrane rafts, but also most crucially discussed [33]. 
Despite all concerns, the differential solubilization of membrane proteins probably depends on their different lipid environment in the membrane. Thus, the enrichment of a protein in DRMs indicates its affinity for presumptive membrane rafts. The most meaningful application of DRM extraction is achieved if there is differential DRM association of a protein before and after a stimulus, thereby linking it to a biological phenomenon [34].

As suggested by the ability of phytosterols to induce phase separation, DRMs could also be isolated from plant material (reviewed in [35]). In the past two years several groups have enlarged the inventory of DRM-associated plant proteins. Morel et al. identified 145 proteins in DRMs of tobacco Bright Yellow-2 (BY-2) cells and analyzed their physicochemical characteristics [36]. They showed that proteins involved in signaling, response to biotic and abiotic stress, cellular trafficking, and cell wall metabolism are overrepresented in the DRM fraction. This resembles the protein composition of DRMs in animal cells [36]. Similarly, DRMs have been isolated from roots of Medicago truncatula and their proteomic and lipid compositions have been extensively analyzed [37]. Interestingly, several proteins belonging to the PM redox system have been found to associate with DRMs (see also below). Recently, Laloi et al. showed how DRM extraction can be combined with genetic approaches [38 $8^{\circ}$. The group isolated DRMs from Arabidopsis cell lines fad 2 and Fad3+ hyperaccumulating 18:1 and 18:3 fatty acids, respectively. The amount of DRMs recovered from the mutant material was $20 \%$ of wild-type levels. This underlines the expected importance of the unsaturation degree of lipid acyl chains in the formation of membrane rafts.

\section{Membrane rafts and mechanisms of subcellular protein localization}

Innate immunity relies on the recognition of pathogenassociated molecular patterns by pattern recognition receptors. Arabidopsis FLAGELLIN SENSITIVE 2 (FLS2) is a receptor-like kinase (RLK) and functions as a pattern recognition receptor that senses bacterial flagellin and activates defense signaling. FLS2 exhibits ligand-induced endocytosis that is abolished in a mutant form of FLS2 that cannot be phosphorylated at a Cterminal threonine residue [39]. Plants expressing this mutant form also have disturbed defense signaling, indicating that receptor endocytosis and defense signaling are interconnected. The presumed membrane compartmentalization leading to receptor endocytosis seems to be required for FLS2 function. Recently, a ligand-induced reduction in the membrane mobility of FLS2 was reported [40]. This finding indicates a change of the environment of the FLS2 receptor upon ligand binding. The cause for the mobility shift could be due to interaction with other proteins, the confinement to less mobile membrane domains, or a combination of both. Indeed, ligand-induced interaction of FLS2 with BRASSINOSTEROID-ASSOCIATED KINASE 1 (BAK1), another RLK, was recently reported [41,42]. It is tempting to speculate that the spatial regulation of FLS2, allowing ligand-induced interactions with BAK1 and endocytosis of the receptor, is partly due to its differential association with membrane rafts. In fact, RLKs are overrepresented in DRMs of plants, indicating their potential in vivo association with membrane rafts $[36,43]$.

The Chlorella kessleri hexose-proton symporter HEXOSE UPTAKE 1 (HUP1) shows a spotty distribution in the PM of Chlorella as well as upon heterologous expression in Saccharomyces cerevisiae. However, in yeast strains lacking ergosterol (erg6) or sphingolipids (lcb1-100), HUP1 is evenly distributed ([19 $\left.{ }^{\circ}\right]$; Figure 2$)$. The catalytic activity of HUP1 is decreased in erg6 cells, indicating that localization of HUP1 to membrane clusters is important for its function. It has been reported that in yeast ergosterol or sphingolipid biosynthesis mutants, proteins that normally localized to membrane rafts fail to associate with them [12]. Furthermore, these mutants are disrupted in membrane polarization at the mating projection [12]. Likewise, the Arabidopsis mutant ortholog of erg6, smerc shows defects in cell polarity [44]. In this mutant, the auxin efflux carrier proteins PIN-FORMED 1 (PIN1) and PIN3 that are normally polarly localized within cells are mislocalized, leading to reduced polar auxin transport [44].

The inwardly rectified $\mathrm{K}^{+}$channel KAT1 is distributed in positionally stable membrane domains in the PM of Nicotiana benthamiana and in a distinct radial pattern in turgid guard cells in Vicia faba [20,21 $\left.{ }^{\circ}\right]$. The radial pattern of KAT1 in guard cells could be attributed to KAT1-cellwall interactions; however, the refinement of KAT1 to membrane domains seems to depend on other factors. Sutter et al. $\left[21^{\circ}\right]$ demonstrated that the membrane pattern and the lateral mobility of KAT1 are severely altered upon overexpression of a dominant-negative fragment (Sp2) of the soluble $N$-ethylmaleimide-sensitive factor attachment receptor (SNARE) protein, SYP121 (Figure 3). While KAT1 is usually present in non-mobile domains in the PM, it is evenly distributed and becomes mobile when coexpressed with Sp2. These data implicate a role for SNAREs in the distribution and behavior of KAT1 at the PM. Furthermore, Sutter $e$ al. showed that the phytohormone abscisic acid selectively triggers KAT1 endocytosis. The exclusion of other proteins from endocytosis requires membrane specialization and the focused recruitment of KAT1 [45]. Whether the regulation of KAT1 is partly achieved through its association with presumptive membrane rafts remains unclear, yet the partial localization of KAT1 in DRMs provides a hint to it $\left[21^{\circ}\right]$. In yeast, the establishment of polar distribution of slowly diffusing PM proteins has been proposed to be achieved through endocytosis [46]. Interestingly, the slow diffusion of proteins is affected in the yeast erg6 mutant, 

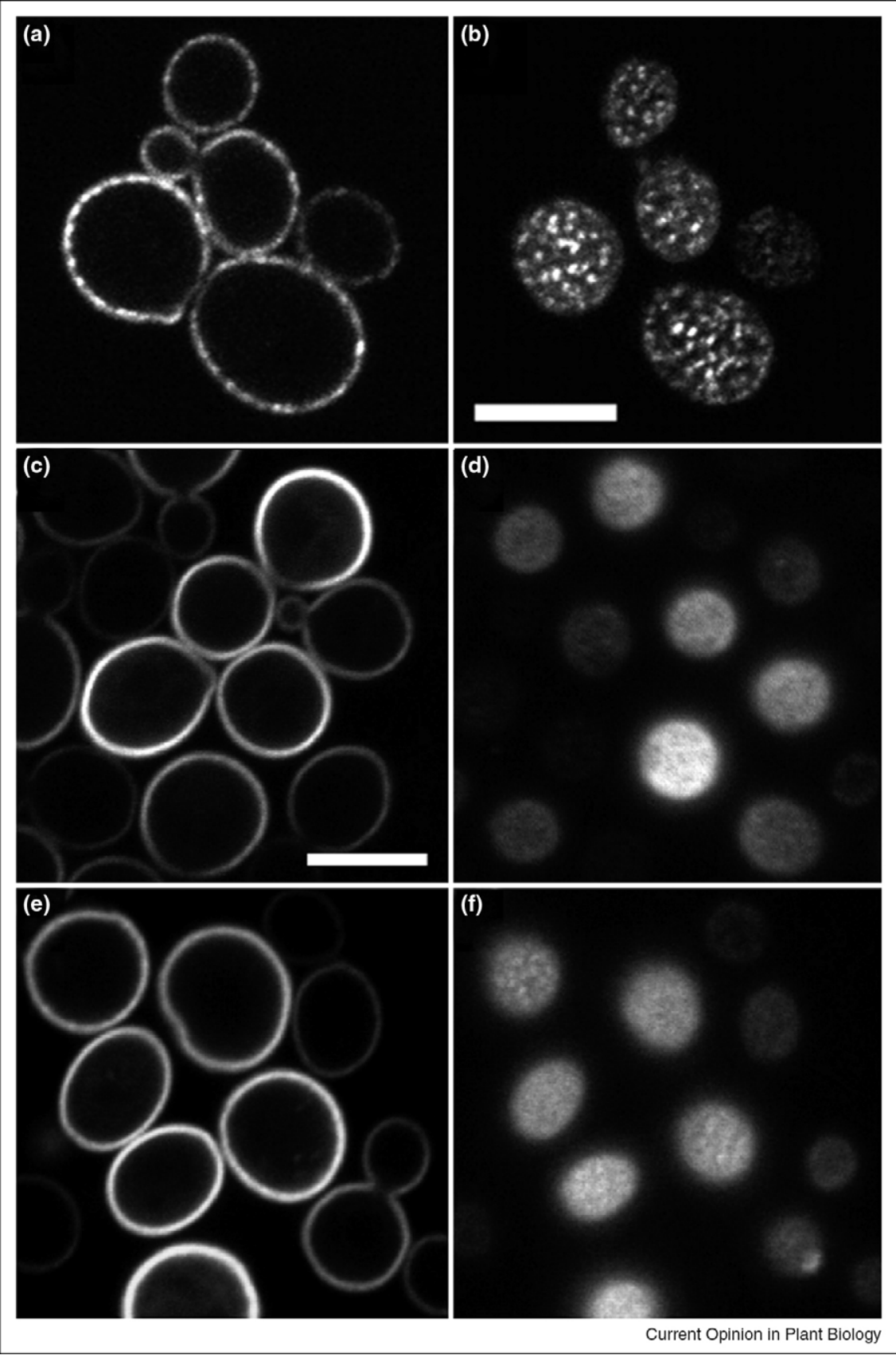

Saccharomyces cerevisiae expressing GFP-tagged Chlorella glucose- $\mathrm{H}^{+}$symporter HUP1. (a and b) HUP1-GFP expressed in Saccharomyces cerevisiae wild type, (c and d) an ergosterol biosynthesis mutant (erg6), and (e and f) a sphingolipid biosynthesis mutant (Icb1-100). (a, c, and e) Confocal cross sections and (b, d, and f) surface views are shown. Size bars, $5 \mu \mathrm{m}$. Newly compiled and modified from [19". Copyright 2006 by the American Society for Microbiology.

in which also the clustering of membrane rafts seems abolished [11,46], Figure 2.

The first publication clearly showing the importance of sterols in the establishment of plant cell polarity revealed a mislocalization of auxin efflux carriers PIN1 and PIN3 in a sterol-deficient mutant accompanied by a reduction in polar auxin transport [44]. Another auxin efflux carrier, PIN2, was already known to colocalize with sterols at the PM and endocytic compartments [47]. Recently, Men et al. reported that the polar localization of PIN2 also depends on correct sterol composition ([48*•]; Figure 4). 
Figure 3

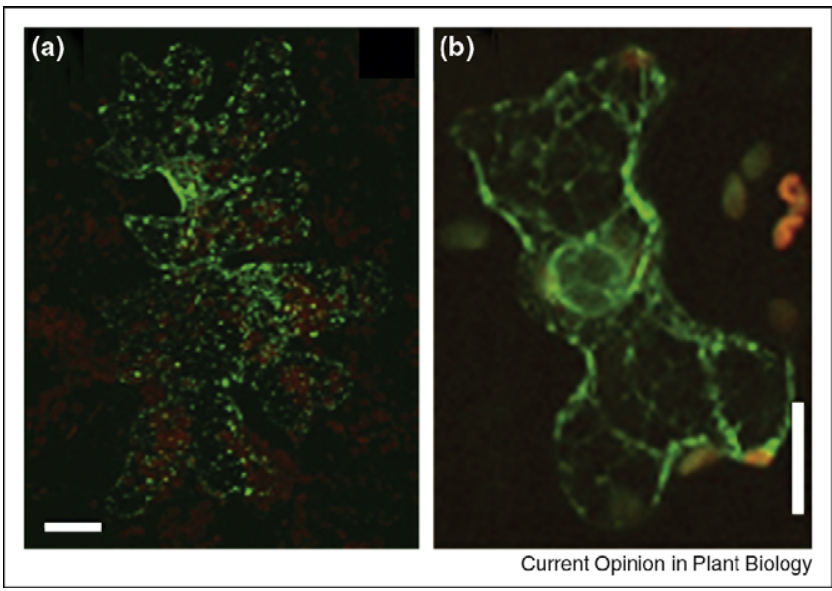

The cytosolic (dominant-negative) Sp2 fragment of tobacco SYP121 selectively suppresses KAT1 $\mathrm{K}^{+}$channel traffic and affects its distribution at the plasma membrane. (a and b) Three-dimensional reconstructions from confocal fluorescence images of tobacco epidermal cells expressing haKAT1-GFP. GFP fluorescence is shown in green, chloroplast fluorescence in red. (a) Expression of haKAT1-GFP only and (b) together with the Sp2 fragment of NtSYP121. Size bars,

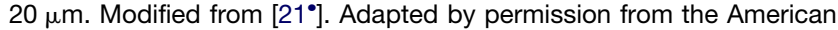
Society of Plant Biologists, copyright 2006.

The sterol biosynthesis mutant cpi1-1 displays aberrant PIN2 localization and a defect in root gravitropsim. The authors elegantly demonstrated that compromised endocytosis, rather than altered lateral mobility or inappropriate PM targeting, is responsible for defects in PIN2 polarity. Still, the generally slow lateral mobility of PIN2 might be important for its polar localization in a scenario where endocytosis regulates the polar distribution of slowly diffusing proteins [46].

It is striking that PIN2 and HUP1, both present in membrane domains, are mislocalized in sterol-deficient mutants that also display compromised endocytosis. Furthermore, KAT1 localization depends on SNAREmediated trafficking of endomembrane compartments. The correct sterol composition, as well as endocytosis and membrane recycling, thus seem to be reoccurring themes in the establishment of plant cell polarity. It would certainly be interesting to investigate whether the elicitor-triggered internalization of FLS2 and the proper localization of KAT1 also depend on a correct sterol composition.

\section{Membrane rafts in biotic interactions}

As mentioned above, it is essential for an organism to react in a spatially and temporally regulated manner to both beneficial and hostile microbes in close proximity. A prominent example for lateral protein heterogeneity is the focal accumulation of a subset of plant PM proteins underneath attempted fungal entry sites. Upon powdery
Figure 4

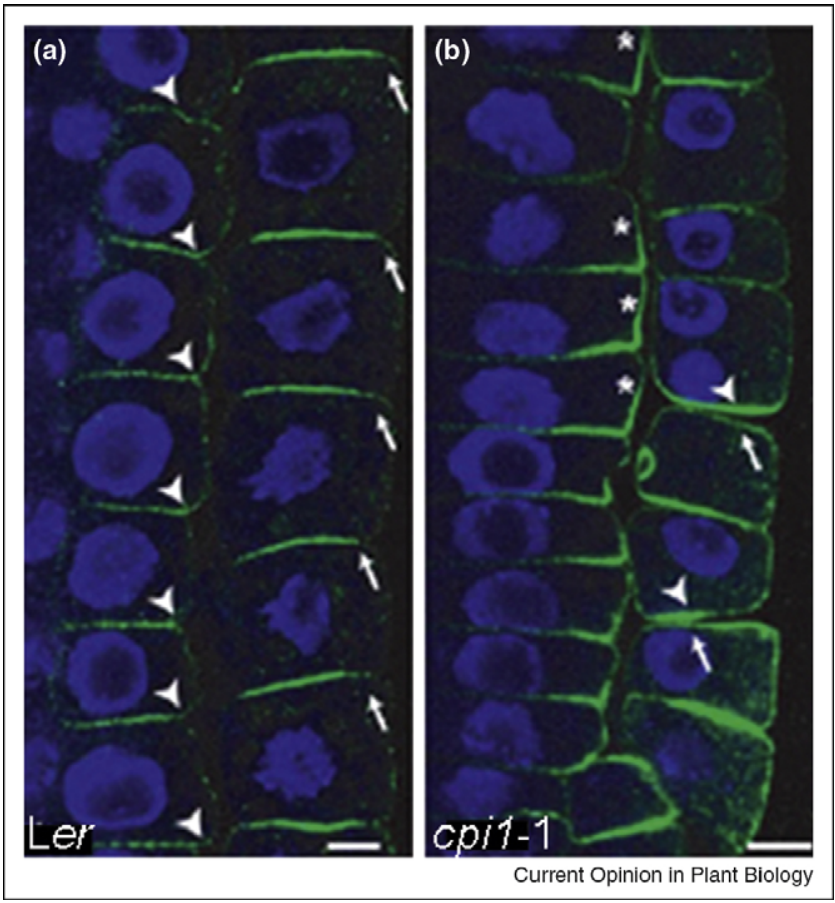

PIN2 polarity requires CPI1 function. (a and $\mathbf{b}$ ) Immunofluorescent PIN2 localization (green) and 4',6-diamidino-2-phenylindole (DAPI) labeling of nuclei (blue) in whole-mount Arabidopsis roots. (a) PIN2 localization at apical epidermal (arrow) and basal cortical (arrowhead) PMs. (b) Apical (arrow) and basal (arrowhead) epidermal as well as basal and lateral (asterisk) cortical PM localization of PIN2 in cpi1-1. Size bars, $5 \mu \mathrm{m}$. Adapted by permission from Macmillan Publishers Ltd: Nature Cell Biology [48*0], copyright 2008.

mildew attack, otherwise evenly distributed fluorophoretagged Arabidopsis and barley polypeptides such as the PM-resident SNAREs SYP121 and ROR2, the heptahelical defense modulator MLO and the cell-death regulator BAX Inhibitor-1 concentrate in stable circular PM domains of $3-10 \mu \mathrm{m}$ diameter [22,23,49]. Formation of this pathogen-triggered multi-polypeptide cluster coincides with a marked staining by the sterol-binding dye filipin, which indicates an accumulation of sterols in these membrane areas [23]. Cellular polarization, comprising focal rearrangement of the cytoskeleton and altered PM organization, is required for the targeted secretion of cargo during the immune responses in plant and animal systems (reviewed in [50]). In this context, membrane rafts have been reported to be involved in receptor-mediated activation of many vertebrate immune cell types, including mast cells, B cells and T cells [51]. Reminiscent of plant SNAREs SYP121 and ROR2 that cluster at powdery mildew attack sites, exocytic SNAREs also accumulate in T cells at the contact site with their respective target cells [52]. Individual human SNARE proteins were found to be enriched in DRMs [53] and in cholesterol-dependent clusters that are distinct from 
typical DRMs [54], suggesting that SNAREs associate with different types of membrane rafts, possibly in an isoform-specific and cell-type-specific manner. Ternary SNARE complexes, the oligomeric SNARE protein assemblies that ultimately mediate vesicle fusion with target membranes, are enriched in DRMs after mast cell stimulation, indicating that membrane rafts might be the preferred sites for secretion [51,54]. This hypothesis is further supported by the finding that cholesterol may promote membrane curvature during endocytic vesicle formation by association with oligomeric cholesterol binding proteins [55].

Though the molecular principles driving large-scale protein assemblies in the PM remain enigmatic, it has recently been suggested that submicrometer-sized SNARE clusters $(50-60 \mathrm{~nm}$ in diameter) originate from self-organization of multiple SNARE molecules, based on weak homophilic protein-protein interactions $\left[56^{\bullet \bullet}\right]$. Individual SNARE molecules dynamically exchange between clusters and the freely diffusing state. SNARE clusters, which are probably functionally important, are probably stabilized by cholesterol $\left[56^{\bullet \bullet}, 57\right]$. It is also conceivable that large-scale PM domains may self-assemble upon a localized stimulus-dependent nucleation event.

Besides polypeptides that are linked to vesicle trafficking/ exocytosis, additional proteins implicated in reactions of plants to biotic stimuli have been reported to reside in DRMs. For example, cytochrome b561, which also focally accumulates at fungal attack sites [23], appears to be an integral part of a PM redox system in the DRMs of Medicago truncatula roots [37]. This complex supposedly establishes the redox balance between cytoplasm and apoplast and might be important for the generation and/or detoxification of reactive oxygen intermediates in plant-microbe interactions [37]. Consistent with this hypothesis, the PM-localized tobacco NADPH oxidase

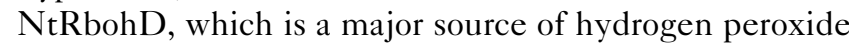
production in pathogen-challenged cells, was found to be recruited to DRMs of BY-2 cells upon stimulation with the fungal elicitor cryptogein [58]. An epitope-tagged variant of the small Rho GTPase NtRac5, a negative regulator of the NADPH oxidase, likewise accumulates in the DRMs of tobacco BY-2 cells [58].

There is also first evidence for a contribution of membrane rafts to a symbiosis-like plant-microbe interaction. Shahollari et al. reported on two genes (At1g13230 and At5g16590) encoding leucine-rich repeat proteins, both transcriptionally upregulated during the interaction of Arabidopsis with the growth-promoting fungus Piriformospora indica [59]. Mutant plants defective in At1g13230 (also designated PIRIFORMOSPORA INSENSITIVE-2, $P i i-2)$ show no growth response to the fungus and no induced transcript accumulation of At5g16590. Interest- ingly, PII-2 and the gene product of At5g16590 are both associated with DRMs, whereas the At5g16590-encoded polypeptide is absent from DRMs of pii-2 plants. Additionally, the authors showed that a mutant in a putative sphingosine kinase also exhibits impaired response to $P$. indica. Taken together, these data nicely demonstrate how the DRM association of a protein can be linked to a biological phenomenon, strengthening the assumption that membrane rafts are important for the function of the protein encoded by At5g16590.

\section{Membrane rafts and tip growth}

Rho GTPases are signaling proteins with an important role in polarization of eukaryotic cells, including directional expansion, asymmetric division and differentiation [17]. They shuttle between an inactive GDP-bound and an active GTP-bound state and regulate the above processes through their specific localization and activation. RAC/ROP (Rho of plant) GTPases, members of the plant Rho GTPase subfamily, are polarly localized at the apical PM of growing root hairs and pollen tubes. Their signaling properties are required for proper tip growth and are regulated by the interaction with regulatory proteins, membrane lipids and $\mathrm{Ca}^{2+}$ [17]. RAC/ROP proteins were shown to be equally distributed between DRMs and Triton X-100 soluble membranes; however, upon activation $\mathrm{RAC} / \mathrm{ROPs}$ partitioned into DRMs [60*0. The authors of this report further investigated the mechanism driving the activation-dependent localization of RAC/ ROPs by studying ROP6, a type-I RAC/ROP. In the GDP-bound state ROP6 is prenylated and localized to soluble membranes, whereas it is reversibly acylated upon activation and then localized to DRMs. A constitutive active mutant form of ROP6 accumulated exclusively in DRMs, while in a double mutant, in which the acylated cysteine is also mutated, the protein again localized to soluble membranes. Similarly, Bloch et al. showed that a constitutive active form of RAC10 accumulated in DRMs and induced malformation of root hairs and leaf epidermal cells, probably through deregulation of endocytosis [61]. The partitioning of activated RAC/ROPs into DRMs strongly suggests a role for membrane rafts in the activation-dependent regulation of RAC/ROPs and establishment of cell polarity. The membrane raft association of proteins of the Ras superfamiliy of small GTPases has been extensively studied in animal cells and similar mechanisms have been suggested for their regulation [62].

Phosphatidylinositol 4,5-bisphosphate $\left(\operatorname{Ptd} \operatorname{Ins}(4,5) P_{2}\right)$ is a signaling lipid that coaccumulates with RAC/ROPs at the apex of growing pollen tubes and root hairs [63]. $\operatorname{PtdIns}(4,5) P_{2}$ has been proposed to function as a RAC/ ROP effector, thereby influencing membrane fusion events. Indeed, PtdIns $(4,5) P_{2}$ has been appointed a role in the regulation of synaptic vesicle endocytosis and exocytosis in animal cells [64]. Furthermore, $\operatorname{PtdIns}(4,5) P_{2}$ seems to promote the activation of RAC/ROPs [17]. In 
animal cells, PtdIns $(4,5) P_{2}$ accumulates at sites of cell surface motility together with a Rho-type GTPase, where it is thought to localize to membrane rafts. $\operatorname{PtdIns}(4,5) P_{2}$ may thereby coordinate membrane dynamics and actin organization as well as integrate signaling [65]. It is therefore tempting to speculate that also in plants the localization of $\operatorname{PtdIns}(4,5) P_{2}$ and RAC/ROPs to membrane rafts provides a mechanism for temporal and spatial organization of signaling and cell polarization. With the help of newly available imaging tools to monitor the $\operatorname{PtdIns}(4,5) P_{2}$ localization at the subcellular level in vivo it will be possible to assess the PtdIns $(4,5) P_{2}$ dynamics in response to various stimuli [66].

Similar to the role of RAC/ROPs in cell polarization through the regulation of actin dynamics and membrane trafficking, barley RACB has been implicated in the modulation of actin reorganization and cell polarity in the interaction of barley with the powdery mildew pathogen [25]. Additionally, in resemblance of PM-resident SNAREs, MLO and BAX Inhibitor-1 (see above), barley ROP-interactive CRIB (Cdc42/Rac-interactive binding) motif-containing protein (RIC) 171 has been found to accumulate underneath attempted fungal entry sites [67]. RICs are plant-specific proteins regulating RAC/ROPs by GTP-dependent interactions. Accordingly, GTP-bound RACB supposedly attracts RIC171 to the PM, suggesting that RACB is present in the GTP-bound state at attempted fungal entry sites.

The focal accumulation of RAC/ROP proteins during polarized growth and cell polarization toward fungal attack sites suggests similar mechanisms involving RAC/ROPs for both processes. Possibly the regulation of NADPH oxidase activity, resulting in the localized generation of reactive oxygen species and the subsequent establishment of a local $\mathrm{Ca}^{2+}$ gradient [68], provides a mechanistic link between both phenomena. Indeed, in both cases also the secretory pathway is polarized toward a distinct cellular region $[17,24]$. It would be interesting to determine whether the signaling lipid $\operatorname{PtdIns}(4,5) P_{2}$ also accumulates underneath fungal attack sites.

\section{Conclusions}

The lateral organization of biological membranes is essential for a large number of biological processes. Remarkably, no consensus on the mechanism underlying membrane domain formation has been reached. The membrane raft hypothesis, which offers a plausible explanation for membrane domain formation, continues to be controversial. However, despite the debate regarding the existence of membrane rafts, researchers mainly agree that large-scale phase separation can occur whether small membrane rafts preexist or not.

In plants, several examples of PM heterogeneity have been described and we have begun to understand the molecular principles for its formation. The degree of fatty acid desaturation, as well as the correct sterol composition in conjunction with endocytosis and membrane recycling appear to be crucial determinants in the lateral organization of plant PMs. Furthermore, accumulating data now point toward conserved molecular mechanisms, including $\mathrm{RAC} / \mathrm{ROP}$ proteins, in polarized growth and cells polarizing toward plant-microbe interaction sites. To further unravel the basis for lateral membrane organization in plants, future research should take advantage of conditionally rescued mutants that may exhibit stronger phenotypes $\left[6^{\bullet \bullet}\right]$. Novel probes, for example, Laurdan, a dye that monitors the order of membranes [10], could also extend the plant toolbox. Combining the analysis of otherwise inaccessible mutants with such new imaging approaches promises to reveal exciting new insights.

\section{Acknowledgements}

We thank Imre Somssich for critically reading this manuscript. Research in the lab of RP is funded by grants from the Max Planck Society and the Deutsche Forschungsgemeinschaft (DFG; SFB670). NFZ is supported by an International Max Planck Research School (IMPRS) fellowship. We thank Rockefeller University Press, Macmillan Publishers Ltd, the American Society for Microbiology, and the American Society of Plant Biologists for the permissions to reproduce previously published figures.

\section{References and recommended reading}

Papers of particular interest, published within the period of review, have been highlighted as:

- of special interest

$\bullet$ of outstanding interest

1. Singer SJ, Nicolson GL: The fluid mosaic model of the structure of cell membranes. Science 1972, 175:720-731.

2. Jacobson K, Mouritsen OG, Anderson RG: Lipid rafts: at a crossroad between cell biology and physics. Nat Cell Biol 2007, 9:7-14.

3. Ikonen E: Cellular cholesterol trafficking and compartmentalization. Nat Rev Mol Cell Biol 2008, 9:125-138.

4. Simons K, Ikonen E: Functional rafts in cell membranes. Nature 1997, 387:569-572.

5. Hancock JF: Lipid rafts: contentious only from simplistic standpoints. Nat Rev Mol Cell Biol 2006, 7:456-462.

6. Pike LJ: Rafts defined: a report on the Keystone Symposium on Lipid Rafts and Cell Function. J Lipid Res 2006, 47:1597-1598.

7. van Meer G, Voelker DR, Feigenson GW: Membrane lipids: where they are and how they behave. Nat Rev Mol Cell Biol 2008, 9:112-124.

8. Kenworthy AK, Nichols BJ, Remmert CL, Hendrix GM, Kumar M, Zimmerberg J, Lippincott-Schwartz J: Dynamics of putative raftassociated proteins at the cell surface. J Cell Biol 2004, 165:735-746.

9. Seminario MC, Bunnell SC: Signal initiation in T-cell receptor microclusters. Immunol Rev 2008, 221:90-106.

10. Gaus K, Chklovskaia E, Fazekas de St Groth B, Jessup W, Harder T: Condensation of the plasma membrane at the site of T lymphocyte activation. J Cell Biol 2005, 171:121-131.

11. Proszynski TJ, Klemm R, Bagnat M, Gaus K, Simons K: Plasma membrane polarization during mating in yeast cells. $J$ Cell Biol 2006, 173:861-866.

12. Bagnat $\mathrm{M}$, Simons $\mathrm{K}$ : Cell surface polarization during yeast mating. Proc Natl Acad Sci U S A 2002, 99:14183-14188. 
13. Gaus K, Le Lay S, Balasubramanian N, Schwartz MA: Integrinmediated adhesion regulates membrane order. J Cell Biol 2006, 174:725-734.

14. Martin SW, Konopka JB: Lipid raft polarization contributes to hyphal growth in Candida albicans. Eukaryot Cell 2004, 3:675-684

15. Marguet D, Lenne PF, Rigneault $\mathrm{H}$, He HT: Dynamics in the plasma membrane: how to combine fluidity and order. $E M B O J$ 2006, 25:3446-3457.

16. Lenne PF, Wawrezinieck $L$, Conchonaud F, Wurtz $O$, Boned $A$ Guo XJ, Rigneault H, He HT, Marguet D: Dynamic molecular confinement in the plasma membrane by microdomains and the cytoskeleton meshwork. EMBO J 2006, 25:3245-3256

17. Kost B: Spatial control of Rho (Rac-Rop) signaling in tipgrowing plant cells. Trends Cell Biol 2008, 18:119-127.

18. Boutte $Y$, Ikeda $Y$, Grebe $M$ : Mechanisms of auxin-dependent cell and tissue polarity. Curr Opin Plant Biol 2007, 10:616-623.

19. Grossmann G, Opekarova M, Novakova L, Stolz J, Tanner W: Lipid

- raft-based membrane compartmentation of a plant transport protein expressed in Saccharomyces cerevisiae. Eukaryot Cell 2006, 5:945-953.

The Chlorella kessleri hexose-proton symporter HEXOSE UPTAKE 1 (HUP1) shows a spotty distribution in the plasma membrane of Chlorella as well as upon heterologous expression in yeast. The localization of HUP1 coincides with sterol-rich membrane domains visualized by filipin In yeast mutants lacking ergosterol or sphingolipids HUP1 is evenly distributed.

20. Homann U, Meckel T, Hewing J, Hutt MT, Hurst AC: Distinct fluorescent pattern of KAT1::GFP in the plasma membrane of Vicia faba guard cells. Eur J Cell Biol 2007, 86:489-500.

21. Sutter JU, Campanoni P, Tyrrell M, Blatt MR: Selective mobility

- and sensitivity to SNAREs is exhibited by the Arabidopsis KAT1 $\mathrm{K}^{+}$channel at the plasma membrane. Plant Cell 2006 18:935-954.

The inwardly rectified $\mathrm{K}^{+}$channel KAT1 is localized to positionally stable domains of $0.5 \mu \mathrm{m}$ in diameter. Disrupting SYP121 function by expressing a dominant-negative Sp2 fragment provides evidence for SNAREmediated traffic of KAT1 and a role for SNAREs in its distribution within domains of the plasma membrane.

22. Assaad FF, Qiu JL, Youngs H, Ehrhardt D, Zimmerli L, Kalde M, Wanner G, Peck SC, Edwards H, Ramonell K et al.: The PEN1 syntaxin defines a novel cellular compartment upon fungal attack and is required for the timely assembly of papillae. $\mathrm{Mol}$ Biol Cell 2004, 15:5118-5129.

23. Bhat RA, Miklis M, Schmelzer E, Schulze-Lefert P, Panstruga R: Recruitment and interaction dynamics of plant penetration resistance components in a plasma membrane microdomain Proc Natl Acad Sci U S A 2005, 102:3135-3140.

24. Kwon C, Neu C, Pajonk S, Yun HS, Lipka U, Humphry M, Bau S, Straus M, Kwaaitaal M, Rampelt $\mathrm{H}$ et al.: Co-option of a default secretory pathway for plant immune responses. Nature 2008 , 451:835-840.

25. Opalski KS, Schultheiss $H$, Kogel KH, Hückelhoven R: The receptor-like MLO protein and the RAC/ROP family G-protein RACB modulate actin reorganization in barley attacked by the biotrophic powdery mildew fungus Blumeria graminis f.sp. hordei. Plant J 2005, 41:291-303.

26. $\mathrm{Xu} X$, London $\mathrm{E}$. The effect of sterol structure on membrane lipid domains reveals how cholesterol can induce lipid domain formation. Biochemistry 2000, 39:843-849.

27. Xu X, Bittman R, Duportail G, Heissler D, Vilcheze C, London E: Effect of the structure of natural sterols and sphingolipids on the formation of ordered sphingolipid/sterol domains (rafts). Comparison of cholesterol to plant, fungal, and diseaseassociated sterols and comparison of sphingomyelin, cerebrosides, and ceramide. $J$ Biol Chem 2001 , 276:33540-33546.

28. Halling KK, Slotte JP: Membrane properties of plant sterols in phospholipid bilayers as determined by differential scanning calorimetry, resonance energy transfer and detergent-induced solubilization. Biochim Biophys Acta 2004, 1664:161-171.
29. Hac-Wydro K, Wydro P, Jagoda A, Kapusta J: The study on the interaction between phytosterols and phospholipids in model membranes. Chem Phys Lipids 2007, 150:22-34.

30. Beck JG, Mathieu D, Loudet C, Buchoux S, Dufourc EJ: Plant sterols in 'rafts': a better way to regulate membrane thermal shocks. FASEB J 2007, 21:1714-1723.

31. Wu R, Chen L, Yu Z, Quinn PJ: Phase diagram of stigmasteroldipalmitoylphosphatidylcholine mixtures dispersed in excess water. Biochim Biophys Acta 2006, 1758:764-771.

32. Brown DA, Rose JK: Sorting of GPI-anchored proteins to glycolipid-enriched membrane subdomains during transport to the apical cell surface. Cell 1992, 68:533-544.

33. Lichtenberg D, Goni FM, Heerklotz H: Detergent-resistant membranes should not be identified with membrane rafts. Trends Biochem Sci 2005, 30:430-436.

34. Lingwood D, Simons K: Detergent resistance as a tool in membrane research. Nat Protoc 2007, 2:2159-2165.

35. Bhat R, Panstruga R: Lipid rafts in plants. Planta 2005, 223:5-19.

36. Morel J, Claverol S, Mongrand S, Furt F, Fromentin J, Bessoule JJ Blein JP, Simon-Plas F: Proteomics of plant detergent-resistant membranes. Mol Cell Proteomics 2006, 5:1396-1411.

37. Lefebvre B, Furt F, Hartmann MA, Michaelson LV, Carde JP, Sargueil-Boiron F, Rossignol M, Napier JA, Cullimore J, Bessoule JJ et al.: Characterization of lipid rafts from Medicago truncatula root plasma membranes: a proteomic study reveals the presence of a raft-associated redox system. Plant Physiol 2007, 144:402-418.

38. Laloi M, Perret AM, Chatre L, Melser S, Cantrel C, Vaultier MN,

- Zachowski A, Bathany K, Schmitter JM, Vallet M et al.: Insights into the role of specific lipids in the formation and delivery of lipid microdomains to the plasma membrane of plant cells. Plant Physiol 2007, 143:461-472.

The authors thoroughly studied the protein content of DRMs from Arabidopsis and leek seedlings and for the first time linked DRM extraction with a genetic approach, showing that fatty acid desaturation is important for DRM formation.

39. Robatzek S, Chinchilla D, Boller T: Ligand-induced endocytosis of the pattern recognition receptor FLS2 in Arabidopsis. Genes Dev 2006, 20:537-542.

40. Ali GS, Prasad KV, Day I, Reddy AS: Ligand-dependent reduction in the membrane mobility of FLAGELLIN SENSITIVE2, an Arabidopsis receptor-like kinase. Plant Cell Physiol 2007, 48:1601-1611.

41. Heese A, Hann DR, Gimenez-lbanez S, Jones AM, He K, Li J, Schroeder JI, Peck SC, Rathjen JP: The receptor-like kinase SERK3/BAK1 is a central regulator of innate immunity in plants. Proc Natl Acad Sci U S A 2007, 104:12217-12222.

42. Chinchilla D, Zipfel C, Robatzek S, Kemmerling B, Nurnberger T, Jones JD, Felix G, Boller T: A flagellin-induced complex of the receptor FLS2 and BAK1 initiates plant defence. Nature 2007, 448:497-500.

43. Shahollari B, Peskan-Berghöfer T, Oelmüller R: Receptor kinases with leucine-rich repeats are enriched in Triton X-100 insoluble plasma membrane microdomains from plants. Physiol Plant 2004, 122:397-403.

44. Willemsen V, Friml J, Grebe M, van den Toorn A, Palme K, Scheres B: Cell polarity and PIN protein positioning in Arabidopsis require STEROL METHYLTRANSFERASE1 function. Plant Cell 2003, 15:612-625.

45. Sutter JU, Sieben C, Hartel A, Eisenach C, Thiel G, Blatt MR: Abscisic acid triggers the endocytosis of the Arabidopsis KAT1 $\mathrm{K}^{+}$channel and its recycling to the plasma membrane. Curr Biol 2007, 17:1396-1402.

46. Valdez-Taubas J, Pelham HR: Slow diffusion of proteins in the yeast plasma membrane allows polarity to be maintained by endocytic cycling. Curr Biol 2003, 13:1636-1640.

47. Grebe M, Xu J, Mobius W, Ueda T, Nakano A, Geuze HJ, Rook MB, Scheres B: Arabidopsis sterol endocytosis involves actin- 
mediated trafficking via ARA6-positive early endosomes. Curr Biol 2003, 13:1378-1387.

48. Men S, Boutte $Y$, Ikeda $Y$, Li X, Palme K, Stierhof $Y-D$,

• Hartmann M-A, Moritz T, Grebe M: Sterol-dependent endocytosis mediates post-cytokinetic acquisition of PIN2 auxin efflux carrier polarity. Nat Cell Biol 2008, 10:237-244.

This study takes us one step further in understanding the role of plant sterols in the establishment of cell polarity and the underlying mechanisms. The authors isolated the sterol biosynthesis mutant cpi1-1, which shows mislocalization of PIN2, probably through altered endocytosis. This finding underlines the importance of endocytic trafficking during the establishment of plant cell polarity.

49. Eichmann R, Dechert C, Kogel K-H, Hückelhoven R: Transient over-expression of barley BAX Inhibitor-1 weakens oxidative defence and MLA12-mediated resistance to Blumeria graminis f.sp. hordei. Mol Plant Pathol 2006, 7:543-552.

50. Kwon C, Panstruga R, Schulze-Lefert P: Les liaisons dangereuses: immunological synapse formation in animals and plants. Trends Immunol 2008, 29:159-166.

51. Puri N, Roche PA: Ternary SNARE complexes are enriched in lipid rafts during mast cell exocytosis. Traffic 2006, 7:1482-1494

52. Das V, Nal B, Dujeancourt A, Thoulouze M-I, Galli T, Roux P, Dautry-Varsat A, Alcover A: Activation-induced polarized recycling targets $T$ cell antigen receptors to the immunological synapse: involvement of SNARE complexes. Immunity 2004, 20:577-588.

53. Chamberlain LH, Burgoyne RD, Gould GW: SNARE proteins are highly enriched in lipid rafts in PC12 cells: implications for the spatial control of exocytosis. Proc Natl Acad Sci U S A 2001, 98:5619-5624.

54. Lang T, Bruns D, Wenzel D, Riedel D, Holroyd P, Thiele C, Jahn R: SNAREs are concentrated in cholesterol-dependent clusters that define docking and fusion sites for exocytosis. EMBO J 2001, 20:2202-2213.

55. Thiele C, Hannah MJ, Fahrenholz F, Huttner WB: Cholesterol binds to synaptophysin and is required for biogenesis of synaptic vesicles. Nat Cell Biol 2000, 2:42-49.

56. Sieber JJ, Willig KI, Kutzner C, Gerding-Reimers C, Harke B,

-• Donnert G, Rammner B, Eggeling C, Hell SW, Grubmuller H et al.: Anatomy and dynamics of a supramolecular membrane protein cluster. Science 2007, 317:1072-1076.

The authors applied a number of sophisticated techniques to unravel the dynamics and architecture of syntaxin 1 membrane clusters. They show that syntaxin 1 aggregates in $50-60 \mathrm{~nm}$ domains containing approximately 75 proteins. This clustering can be explained by self-association of syntaxin 1 based on weak homophilic protein-protein interactions.

57. Lang T: SNARE proteins and 'membrane rafts'. J Physiol 2007, 585:693-698.

58. Mongrand S, Morel J, Laroche J, Claverol S, Carde JP, Hartmann MA, Bonneu M, Simon-Plas F, Lessire R, Bessoule JJ:
Lipid rafts in higher plant cells: purification and characterization of Triton X-100-insoluble microdomains from tobacco plasma membrane. J Biol Chem 2004 279:36277-36286

59. Shahollari B, Vadassery J, Varma A, Oelmüller R: A leucine-rich repeat protein is required for growth promotion and enhanced seed production mediated by the endophytic fungus Piriformospora indica in Arabidopsis thaliana. Plant J 2007, 50:1-13.

60. Sorek N, Poraty L, Sternberg H, Bar E, Lewinsohn E, Yalovsky S:

- Activation status-coupled transient $S$ acylation determines membrane partitioning of a plant Rho-related GTPase. Mol Cell Biol 2007, 27:2144-2154.

In this study the authors elegantly investigated the transient acylation of ROP6 upon GTP binding, which leads to the accumulation of ROP6 in DRMs. In the GDP-bound state ROP6 is prenylated and localized to soluble membranes.

61. Bloch D, Lavy M, Efrat Y, Efroni I, Bracha-Drori K, Abu-Abied M Sadot E, Yalovsky S: Ectopic expression of an activated RAC in Arabidopsis disrupts membrane cycling. Mol Biol Cell 2005, 16:1913-1927.

62. Abankwa D, Gorfe AA, Hancock JF: Ras nanoclusters: molecular structure and assembly. Semin Cell Dev Biol 2007, 18:599-607.

63. Cole RA, Fowler JE: Polarized growth: maintaining focus on the tip. Curr Opin Plant Biol 2006, 9:579-588.

64. Cremona O, De Camilli P: Phosphoinositides in membrane traffic at the synapse. J Cell Sci 2001, 114:1041-1052.

65. Golub $\mathrm{T}$, Caroni $\mathrm{P}$ : $\mathbf{P I}(\mathbf{4}, \mathbf{5}) \boldsymbol{P}_{\mathbf{2}}$-dependent microdomain assemblies capture microtubules to promote and control leading edge motility. J Cell Biol 2005, 169:151-165.

66. van Leeuwen W, Vermeer JE, Gadella TW Jr, Munnik T: Visualization of phosphatidylinositol 4,5-bisphosphate in the plasma membrane of suspension-cultured tobacco BY-2 cells and whole Arabidopsis seedlings. Plant J 2007, 52:1014-1026.

67. Schultheiss H, Preuss J, Pircher T, Eichmann R, Hückelhoven R: Barley RIC171 interacts with RACB in planta and supports entry of the powdery mildew fungus. Cell Microbiol 2008 doi: 10.1111/j.1462-5822.2008.01167.x

68. Foreman J, Demidchik V, Bothwell JH, Mylona P, Miedema $\mathrm{H}$ Torres MA, Linstead P, Costa S, Brownlee C, Jones JD et al.: Reactive oxygen species produced by NADPH oxidase regulate plant cell growth. Nature 2003, 422:442-446.

69. Babiychuk E, Bouvier-Nave P, Compagnon V, Suzuki M,

-. Muranaka T, Van Montagu M, Kushnir S, Schaller H: Allelic mutant series reveal distinct functions for Arabidopsis cycloartenol synthase 1 in cell viability and plastid biogenesis. Proc Natl Acad Sci U S A 2008, 105:3163-3168.

The authors rescued an otherwise lethal sterol-biosynthesis mutant employing a CRE/loxP recombination-dependent strategy. Lethal cas1-2 mutants were complemented with CAS1 cDNA, which can be conditionally excised by the CRE recombinase. 